\title{
Interaction between Wnt/ß-catenin pathway and microRNAs regulates epithelial-mesenchymal transition in gastric cancer (Review)
}

\author{
CUNEN WU ${ }^{1,2^{*}}$, YUWEN ZHUANG ${ }^{1,2^{*}}$, SHAN JIANG ${ }^{3}$, SHENLIN LIU $^{1}$, JINYONG ZHOU $^{1}$, \\ JIAN WU ${ }^{1}$, YUHAO TENG ${ }^{1,2}$, BAOMEI XIA ${ }^{1,2}$, RUIPING WANG $^{1}$ and XI ZOU ${ }^{1}$ \\ ${ }^{1}$ Department of Oncology, The Affiliated Hospital of Nanjing University of Chinese Medicine; \\ ${ }^{2}$ The First Clinical Medical College, Nanjing University of Chinese Medicine, Nanjing, \\ Jiangsu 210029, P.R. China; ${ }^{3}$ Department of Bioscience, Faculty of Bioscience, \\ Nagahama Institute of Bio-Science and Technology, Nagahama, Shiga 526-0829, Japan
}

Received February 6, 2016; Accepted March 15, 2016

DOI: $10.3892 / \mathrm{ijo} .2016 .3480$

\begin{abstract}
Gastric cancer (GC) is the third primary cause of cancer-related mortality and one of the most common type of malignant diseases worldwide. Despite remarkable progress in multimodality therapy, advanced GC with high aggressiveness always ends in treatment failure. Epithelialmesenchymal transition (EMT) has been widely recognized to be a key process associating with GC evolution, during which cancer cells go through phenotypic variations and acquire the capability of migration and invasion. Wnt/ $\beta$-catenin pathway has established itself as an EMT regulative signaling due to its maintenance of epithelial integrity as well as tight adherens junctions while mutations of its components will lead to GC initiation and diffusion. The E-cadherin $/ \beta$-catenin complex plays an important role in stabilizing $\beta$-catenin at cell membrane while disruption of this compound gives rise to nuclear translocation of $\beta$-catenin, which accounts for upregulation of EMT biomarkers and unfavorable prognosis. Additionally, several microRNAs positively or negatively modify EMT by reciprocally acting with certain target genes of $\mathrm{Wnt} / \beta$-catenin pathway in GC. Thus, this review centers on the strong associations between $\mathrm{Wnt} / \beta$-catenin pathway and microRNAs during alteration of EMT in GC, which may induce advantageous therapeutic strategies for human gastric cancer.
\end{abstract}

\footnotetext{
Correspondence to: Professor Ruiping Wang or Professor Xi Zou, Department of Oncology, The Affiliated Hospital of Nanjing University of Chinese Medicine, 155 Hanzhong Road, Nanjing, Jiangsu 210029, P.R. China

E-mail: wrp61@163.com; ruipingwang61@hotmail.com

E-mail: zxvery@126.com

*Contributed equally
}

Key words: gastric cancer, epithelial-mesenchymal transition, Wnt/ $\beta$ catenin pathway, microRNA, targeted therapy

\section{Contents}

1. Introduction

2. Epithelial-mesenchymal transition and gastric cancer

3. Wnt/ $\beta$-catenin pathway and gastric cancer

4. Interaction between EMT and Wnt/ $\beta$-catenin signaling in gastric cancer

5. MicroRNA-mediated EMT regulatory network in gastric cancer through Wnt pathway

6. Promising targeted therapy in gastric cancer

7. Conclusions and perspectives

\section{Introduction}

Gastric cancer, one of the most common malignancies and causes almost one million new cases each year in the world (1). To make matters worse, despite decreasing incidence over the recent decades, GC remains the third leading cause of global cancer-related death preceded by lung and liver cancer and maintains the highest morbidity in Eastern Asia $(2,3)$. The main reasons for failure and mortality of gastric cancer are cell infiltration and metastasis. Metastatic cancer cells fractionally retain their epithelial properties and obtain mesenchymal characteristics which give them the ability to invade or distract. E-cadherin is a major protein prominently required for cell-cell junctions and polarity, whose loss has been established to be the principal event in metastatic progression and tumor invasion. Moreover, downregulation of E-cadherin might induce the occurrence of EMT, a multistage process giving rise to the transformation of polar epithelial cells to mesenchymal phenotype and authorizes cell migration as well $(4,5)$.

Two decades ago, Heuberger and Birchmeier made the first ascertainment to expound that the presence of $\mathrm{E}$-cadherin $/ \beta$ catenin complex allows for steady cell junctions (6). $\beta$-catenin, one key protein in Wnt signaling, separates from the degradation complex and then accumulates in the cytoplasm, translocates into nucleus subsequently to sensitize the Wnt pathway during 
carcinoma progression. In the presence of downregulation of E-cadherin and Wnt signals activation, both cytoplasmic and nuclear levels of $\beta$-catenin increase where it interacts with cell growth and EMT $(7,8)$. MicroRNAs are small noncoding molecules with 19-25 nucleotides that bind to 3'UTR of target mRNAs with complementary base pairing to inhibit their translation or speed up decomposition. Latest experiments investigated either suppressive or promotive function of crosstalk between miRNAs and certain signaling pathways during EMT and metastasis, in spite of this, little attention has been paid to the interplay between Wnt/ $\beta$-catenin signaling and miRNAs in regulating EMT in gastric cancer (9). Taking efforts to comprehend the mechanism of Wnt adjustment by miRNAs may benefit our perspectives of reversing the course of EMT or potential therapeutic targets in GC. Therefore, this review focuses on crosstalk between microRNAs and Wnt pathway in the course of modulating EMT in gastric cancer.

\section{Epithelial-mesenchymal transition and gastric cancer}

Epithelial-mesenchymal transition, firstly put forward by Greenburg and Hay in 1982 (10), has been considered to be the feature of embryonic development. However, emerging evidence has indicated that the occurrence of EMT is critical for the initiation and progression of diverse tumors. An EMT process is distinguished by loss of E-cadherin and it creates profound phenotypic alterations through which epithelial cells with apico-basal polarity convert into front-rear polarity to gain mesenchymal characteristics as well as the capacity of migration, invasion and apoptotic resistance (11) (Fig. 1). The epithelial cells going through EMT become invasive and then migrate into distant tissues where they experience a reverting mechanism mesenchymal-epithelial transition (MET), afterward these cells retrieve membrane junctions and form metastatic focus (12). EMT is characterized by the mutative expression of three distinct families of protein consist of cadherins (cell membrane surface protein), Vimentin (cytoskeletal protein) and transcription factors including Snail, Twist, and ZEB $(13,14)$.

E-cadherin, encoded by CDH1, interacts with $\alpha, \beta, \gamma$ and P120-catenin to constitute E-cadherin/catenin complex that plays a critical role in adherens junctions between epithelial cells (15). Loss of E-cadherin on account of promoter hypermethylation has been widely considered to correlate with GC aggressiveness and metastasis as well as unfavorable prognosis of patients $(16,17)$. Hansford et al $(18)$ reported that HDGC families, particularly over the age of 80 , may be best defined by mutations in $\mathrm{CDH} 1$ and different clinical ramifications, and that $\mathrm{CDH} 1$ mutation might provide more accurate estimations of age-connected risk of GC. Nevertheless, Li et al (19) demonstrated that CDH1 promoter hypermethylation probably contributes to E-cad reduction in sporadic instead of hereditary gastric cancer. It is widely acknowledged that in the presence of deviant EMT, E-cadherin switches to N-cadherin which is generally distributed in neural and mesenchymal cells and has ability to expedite the degeneration of stroma as well as cell movement. Jun et al suggested that median survival is significantly correlated with $\mathrm{N}$-cadherin expression in cases of gastric cancer with brain metastasis (20). Similarly, Kamikihara et al (21) performed a genetic association study in
146 gastric cancer patients to analyze the relationship between $\mathrm{N}$-cadherin expression and clinicopathological features. The result exhibited that $\mathrm{N}$-cadherin expression positively correlates to hematogenous relapse while negatively connects to patients' postoperative outcome independent of E-cadherin expression, even if $\mathrm{N}$-cadherin has no association with tumor histology or lymphatic invasion.

Vimentin, a hallmark of interstitial cells, is known to be tightly associated with the potential of tumor cell invasiveness and migration. Vimentin expresses obviously higher diffuse type than intestinal in patients with gastric adenocarcinoma and it might be a feasible mark of relapse, far metastasis or even decreased survival (22). Shirahata et al (23) recommended we use quantitative methylation-specific PCR (qMSP), a sensitive technique that can catch as few as one methylated gene copy out from 1,000 unmethylated ones, to detect promoter methylation in the serum of GC patients. In addition, they recorded a high correlation between Vim methylation and gastric carcinogenesis.

The zinc finger protein Snail consists of three members containing Snail1, Snail2 (Slug) and Snail3 (Smuc), among which Snaill and Snail2 act synergistically in cases of gastric tumor. Snail is initially recognized to facilitate EMT by way of distinguishing E-box motifs in CDH1 to inhibit E-cadherin, whereas growing indication has inferred the mechanism involves not only downregulation of claudins, occludins, cytokeratins but also upregulation of fibronectin and vitrinectin (24). Besides, the involvement of NF- $\kappa \mathrm{B}-$ Snail-E-cadherin axis or CCR7 pathway in induction of EMT through upregulating Snail signaling proved in GC both implied the decisive role of Snail explicitly $(25,26)$. Furthermore, Shin et al (27) reported that Snail could be a prognostic predictor of gastric cancer due to its strong interrelationship with tumor progression, lymph node metastasis, lymphovascular or perineural invasion. Twist protein is a member of the basic/helix-loop-helix transcription factor family and binds selectively to the E-box consensus sequence to regulate target genes such as E-cadherin (28). A series of experiments have illustrated the considerable function of Twist as an oncogene in vivo, suggestive of its potency to be a target for the treatment of gastric carcinoma. Liu et al (29) discovered that Twist is expressed much higher in gastric cancer tissues compared to adjacent normal tissues at mRNA and protein levels by means of RT-PCR and western blotting. Likewise, abnormal Twist expression related strongly to lower 5-year survival rates in patients of GC in the $\mathrm{Ru}$ et al study, yet, they emphasized that the significance disappears in cases of stage IV (30). Various examinations in vitro collaboratively implied the carciogenesis of Twist. GC cells transfected with Twist 1 accelerated cell cycle progression by recruiting p300 to enhance FoxM1 gene expression, whereas those treated with Twist siRNA reversely experienced obvious cell cycle arrest at G0/G1 phase, apoptosis induction as well as decreased ability of proliferation and invasion $(31,32)$. Apart from this, Twist was proved to be a pivotal gene of Helicobacter pyloritriggered GC cell stemness while decreased after $H$. pylori eradication (33), reminding us to excavate therapeutic targets against gastric cancer via Twist-dependent mechanism. ZEB family members, ZEB1 and ZEB2, are transcriptional factors suppressing E-cadherin, and they play a decisive role in tumor 

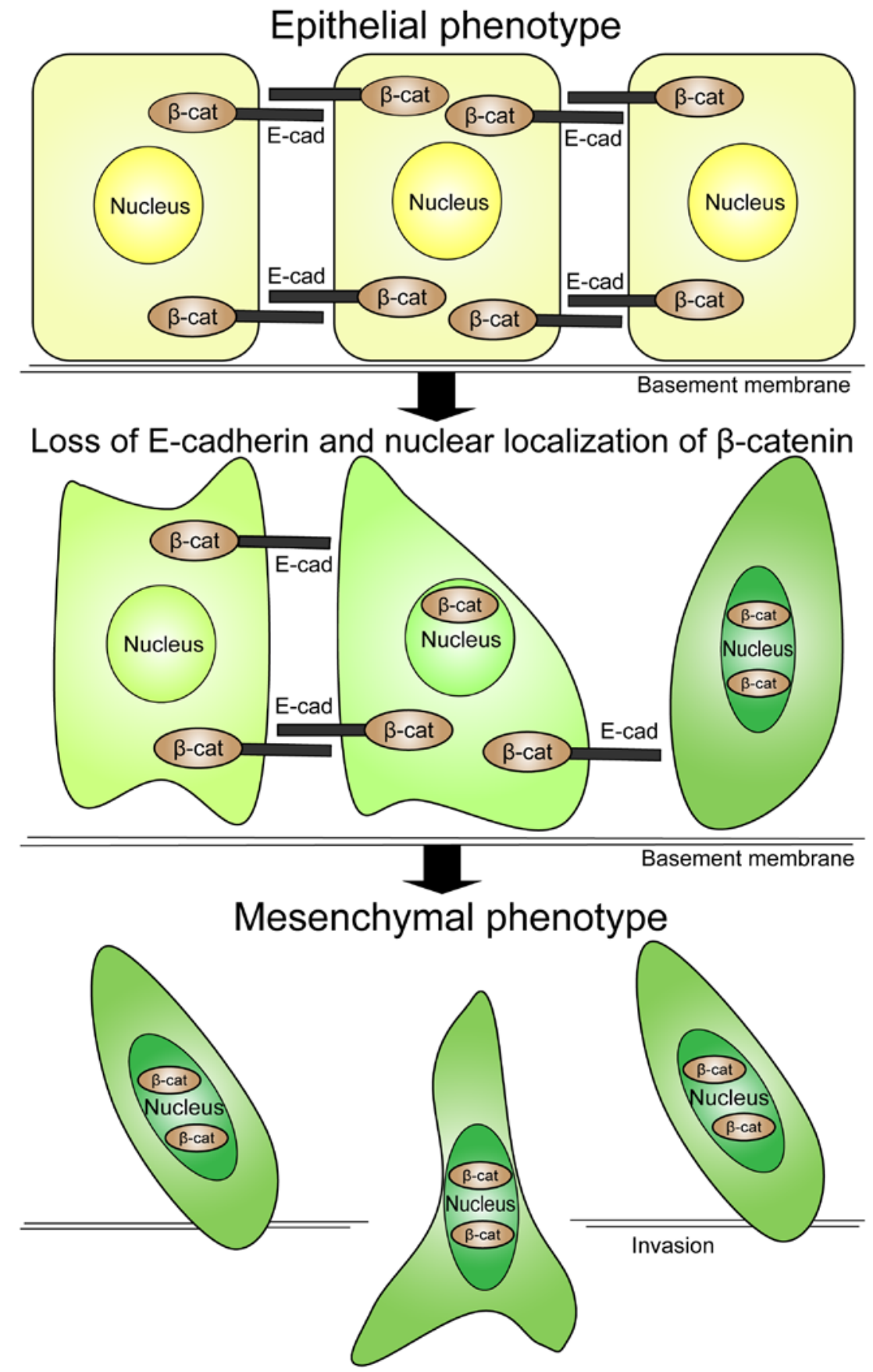

Figure 1. Loss of E-cadherin is generally accepted to be a hallmark event of the EMT process, during which epithelial cells undergo profound phenotypic modification and lack of polarity to turn into mesenchymal cells, reducing contact with surrounding cells or matrix as well as acquiring the ability of migration and invasion.

metastasis. Substantial evidence showed that intense expression of ZEB1 associates with both primary gastric cancer and peritoneal diffusion which predicted earlier recurrence as well as lower disease-free survival rate $(34,35)$. Murai et al (36) classified $116 \mathrm{GC}$ patients into epithelial or mesenchymal group through Vimentin/E-cadherin ratio (V/E), as they pointed, the mesenchymal group with high $\mathrm{V} / \mathrm{E}$ ratio indicated an EMT status and carried anomalous ZEB1 expression, suggesting aggressive clinicopathological characteristics and poorer survival. Moreover, less AGS cells passed through the Transwell chamber which signified reduced capability of invasion in response to transfection with ZEB1 siRNA (37).
ZEB2 can also bind to the E-box of E-cadherin gene promoter similarly to the Snail family. In light of the exploration by Dai et al, ZEB2 was closely tied to poor clinicopathological parameters such as depth of invasion, lymph node metastasis, TNM stage along with higher expression of matrix metalloprotease (MMP) family (38). A recent study from Korea on brain metastatic gastric adenocarcinoma argued that four of eight primary GC cases represent evident nuclear ZEB2 expression. Nevertheless, 3 cases showed decreased nuclear ZEB2 in brain metastatic gastric cancer samples corresponding to the consequence of brain metastatic adenocarcinoma cell research, which might result from the complex mechanisms 
of brain metastasis or small sample size due to low incidence (39). However, this finding might support the theory of MET process that anchors circulating cancer cells to metastatic organs, although the effect of this in metastatic dissemination is still under debate (40).

'Cancer stem cell' was first put forward by Reya et al in 2011 and they protested conspicuous parallels between stem cells and cancer cells through the discovery of the origination of tumors from normal stem cells as well as analogical signaling pathways in regulating self-renewal of either stem cells or cancer cells (41). The existence of gastric cancer stem cells (GCSCs) was subsequently demonstrated and investigators isolated GCSCs through employing surface markers involving CD44/CD24 (42), EpCAM/CD44 (43), CD90 (44), CD44/CD54 (45), and CD47 (46). To date, increasing number of research studies strongly suggest that, GC cells acquire cancer stem cell (CSC) properties including self-renew, prevention of apoptosis, chemotherapy or irradiation resistance when undergoing EMT. Yang et al (47) obtained viable GCSCs from SGC7901 by use of serum-free and epidermal growth factor (EGF) containing medium. Whereafter, they detected greater empowerment of invasion and metastasis remained with decreased E-cadherin, but increased Vimentin expression of these cells that intensively implied the occurrence of EMT. H. pylori has been publicly accepted to be one of the pathogenic factors for gastric cancer while a number of studies have testified that $H$. pylori generates stemness maintenance of GC cells or clinical samples via induction of epithelial-mesenchymal transition simultaneously $(33,48)$. Therefore, investigators advocated to combine CSC with EMT markers, specifically E-cadherin, Vimentin and CD44, as a key predictor of GC recurrence $(49,50)$. Additionally, further exploration ought to be carried out to illuminate the impact of EMT on gastric cancer progression, which could bring about more accurate cancer therapies.

\section{Wnt/ $\beta$-catenin pathway and gastric cancer}

The Wnt pathway named from wingless and Int-1, plays a foremost role in cell proliferation, differentiation, adhesion, migration and stem cell self-renewal. This signaling could be divided into two categories through whether it is $\beta$-catenin dependent (canonical) or $\beta$-catenin independent (non-canonical). Normally, cytoplasmic $\beta$-catenin is trussed up by the destruction complex made up of Axin, adenomatous polyposis coli (APC), casein kinase $1 \alpha(\mathrm{CK} 1 \alpha)$ and glycogen synthase kinase $3 \beta$ (GSK3 $\beta$ ), which is distinguished by the E3 ubiquitin ligase $\beta$-TrCP and the proteosomal degradation ultimately (51). The steady state is destroyed when secreted Wnt ligands bind to receptors on the surface of cell membrane mainly refer to as Frizzled (Fz)/low-density lipoprotein receptor-related protein 5/6 (LRP5/6) receptor compound. Afterwards, Dishevelled (Dsh) protein gets phosphorylated and forms a complex with Axin, which subsequently binds GSK-3 $\beta$ so as to block its activation (52). This association further results in decomposition of the degradation complex and $\beta$-catenin gathers in the cytoplasm (53). Cumulative $\beta$-catenin translocates into the nucleus, which has been generally acknowledged to be the principle event of canonical Wnt pathway activation, next interacts with TCF/LEF to compose a complex of activators and accordingly set off the transcription of target genes of $\mathrm{Wnt} / \beta$-catenin signaling including $\mathrm{c}-\mathrm{Myc}$, cyclinD1, and MMP-7 (54) (Fig. 2).

Several mutated components among the members of canonical Wnt signaling, which further result in aberrant activation of the $\mathrm{Wnt} / \beta$-catenin pathway, play a paramount role in malignant transformation and invasiveness of gastric cancer. For instance, human Wnts has been proved to participate in stomach tumor progression in autocrine or paracrine manner. Upregulation of Wnt-1 ligand was reported to be capable of either maintaining the stemness of GCSC or promoting the advanced stages of gastric cancer $(55,56)$. In addition, augmented Wnt-2 might simultaneously result in cytoplasmic $\beta$-catenin accumulation and nuclear localization in both intestinal- and diffuse-type gastric carcinoma, which was positively associated with stomach cancer formation as well as tumor invasion or dissemination (57). Kurayoshi et al (58) argued that Wnt-5a gives rise to stimulating cell migration and invasion in gastric cancer cells by means of activating focal adhesion kinase and the small GTP-binding protein Rac. Previous studies stated that upregulation of Wnt10A induced by tumor necrosis factor $\alpha(\mathrm{TNF} \alpha)$ and Helicobacter pylori (H. pylori) infection might shed light on gastric carcinogenesis (59). Saitoh et al (60) confirmed the truth of the ability of Wnt10B to trigger the development of gastric cancer. Moreover, both Wnt10A and Wnt10B in GC was tied up with activation of the $\beta$-catenin-TCF signaling pathway. CDH1 gene was verified to encode E-cadherin so as to sustain epithelial cell-cell adhesion and suppress tumor invasion while germline gene alterations of CDH1 had a causative role in $\sim 30-50 \%$ of hereditary diffuse gastric cancer (HDGC) $(61,62)$. In particular, non-mutated (second) $\mathrm{CDH} 1$ allele resulting chiefly from promoter hypermethylation was likely to disintegrate cell adherens junctions and was privy to loss of cell polarity, subsequent to which, $\beta$-catenin was activated and HDGC initiated $(63,64)$. Intestinespecific transcription factor CDX2 has been found responding to the generation of gastric adenocarcinoma from intestinal metaplasia, which revealed nuclear staining of $\beta$-catenin and APC gene mutations simultaneously $(65,66)$.

Exon 3 of CTNNB1, where almost all the $\beta$-catenin mutations reported in human cancers are localized, is widely accepted to encode serine-threonine phosphorylation sequence for GSK3 $\beta$ that regulates destruction of $\beta$-catenin through the ubiquitin-proteasome pathway. Multiple experiments provided evidence indicating that mutations in exon 3 of CTNNB1 precipitates not only continuous activation of Wnt pathway but also multistep stomach carcinogenesis $(67,68)$. Galectin-3, whose mutation locates at position 191, has been widely recognized equipped to substitute proline to histidine (gal-3H64), followed by increased nuclear accumulation of $\beta$-catenin as well as promotion of TCF transcription during gastric cancer evolvement (69). RNF43, a transmembrane E3 ligase, has been shown to eliminate Wnt receptors on cell surface in order to restrain Wnt signaling $(70,71)$. Of note, according to a study from Korea, RNF43 gene might harbor mutational regional intratumoral heterogeneity (ITH), which could be accountable for tumorigenesis of GC (72). Lu et al (73) discussed that enhancer of zeste homolog 2 (EZH2) has ectopic expression in gastric cancer tissues. In a mechanistic manner, repressing CXXC finger protein 4 (CXXC4), a protein 


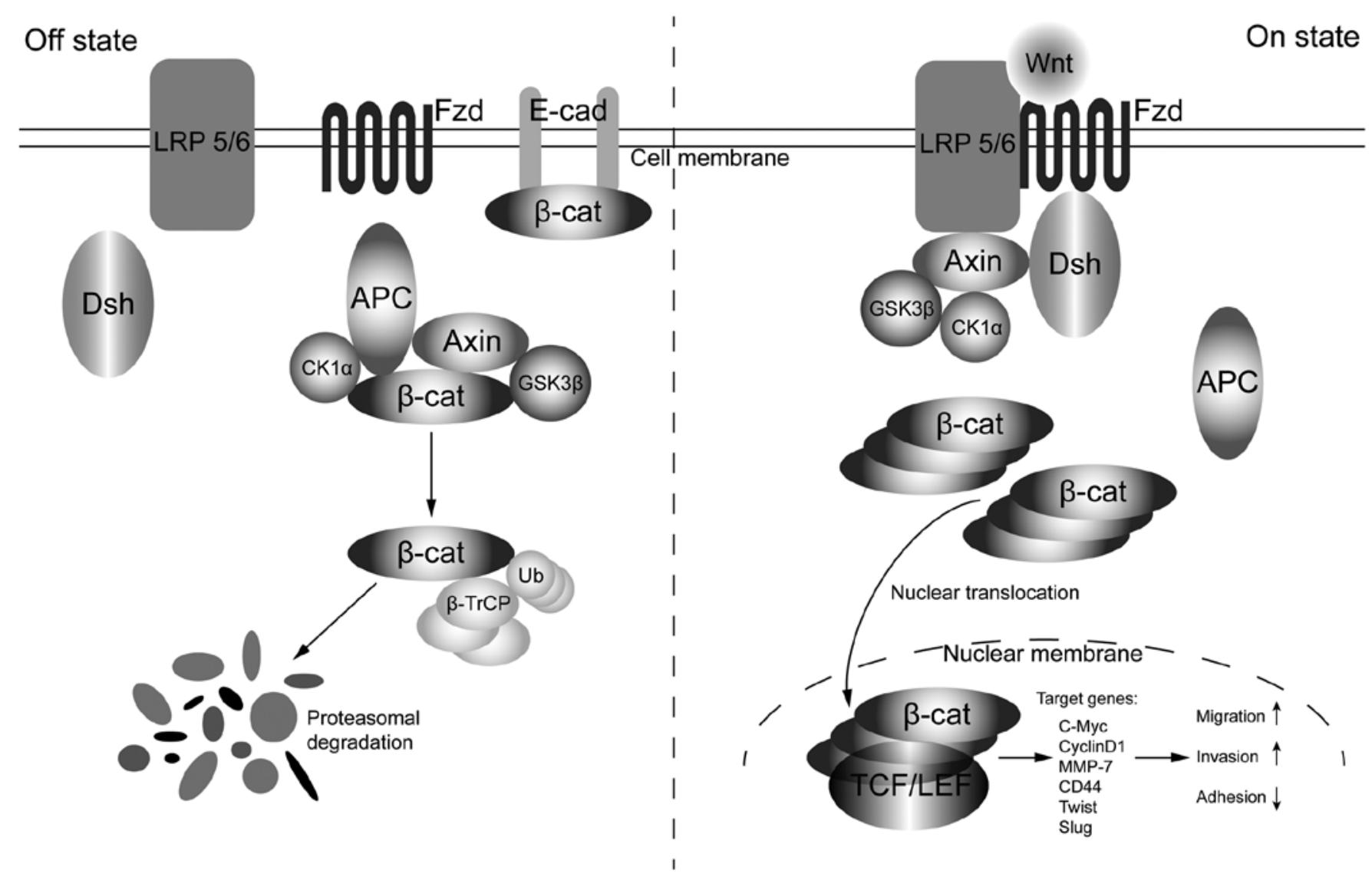

Figure 2. (Off state) In the absence of a Wnt ligand, cytoplasmic $\beta$-catenin is restrained by the degradation compound composed of Axin, APC, CK1 $\alpha$ and GSK3 $\beta$, which is discerned by the E3 ubiquitin ligase $\beta$-TrCP and sequentially is ubiquitinated. (On state) In the presence of a Wnt ligand, it binds to Fzd/ LRP5/6 receptor complex on the surface of the cell membrane. Afterwards, Dsh protein is recruited and constitutes a complex with Axin, which binds GSK-3 $\beta$ and CK1 $\alpha$ so as to emancipate $\beta$-catenin later on. This sequence of steps further leads to cytoplasmic accumulation of $\beta$-catenin subsequent to decomposition of the degradation complex. Stabilized $\beta$-catenin translocates into the nucleus and take part in interplay with TCF/LEF to trigger target genes including c-Myc, cyclin D1, and MMP-7.

stabilize the degradation complex of $\beta$-catenin, was necessary for the contribution of EZH2 to GC. Many investigations in GC cases identified crucial association between Actin-binding protein anillin (ANLN) expression and clinical features while transcription factor analysis demonstrated the possession of TCF binding sequence in ANLN gene promoter. Interestingly, ANLN manifested higher expression among proliferative type gastric tumors compared to invasive or metabolic types (74). Overexpression of cadherin-17 (CDH17), frizzled (FzE3), Yin Yang 1 (YY1), porcupine (PPN) has all been discovered to account for Wnt motivation, together with cell growth in gastric cancer (75-78).

Besides, loss of Wnt repressor function has been authenticated answerable to gastric cancer conformation. Dickkopf (DKK) family has been shown to bring about Wnt signal inhibition by way of binding to LRP5/6 while epigenetic silence of DKK genes is frequently detected in GC specimens. As an example, DKK-1 was found to be capable of antagonizing $\mathrm{Wnt} / \beta$-catenin pathway as well as weakening the self-renewing ability of cancer stem-like cells (CSLCs) isolated from MKN-45 cell line (79). Furthermore, DKK-3 is known as negative regulator of $\mathrm{Wnt}$, whose reduced expression primarily is due to promoter methylation involved in poor outcome of gastric carcinoma (80). Than et al (81) reported that administration of adenovirus vector carrying REIC/DKK-3 (Ad-REIC/DKK-3) can inhibit scirrhous gastric carcinoma (SGC) both in vitro and in nude mice by boosting apoptosis and recruitment of NK cells. On the contrary, despite DKK-1 or DKK-3 being Wnt suppressors, these protein have been detected upregulated in GC as well as significantly correlated to pT-stage and UICC stage $(82,83)$, revealing that DKKs could have potential in oncogenesis of gastric cancer rather than as tumor inactivators, which might be explained either as a negative feedback loop in response to initial $\mathrm{Wnt} / \beta$-catenin signaling activation or mediation by non-canonical Wnt pathways (84). Shin et al (85) sought to assess the role of secreted frizzled-related proteins (SFRPs) as antagonist of Wnt pathway and confirmed that aberrant methylation of SFRPs is the major mechanism by which Wnt signaling is activated in human GC cells. Intestinal-type gastric cancer patients exhibit somatic mutation in germline APC, a pivotal Wnt inhibitor, whose locus at the chromosome sequence 5q21-22 is frequently lost in heterozygosity (86). APC gene deletion was chiefly collected in advanced GCs, suggesting that it might take place in the progression but not initiation of tumors and had profound influence on aggressiveness (87). Axin is the scaffold protein in $\beta$-catenin degradation compound while both germline and somatic 
alterations in Axin1 or Axin2 genes have been affirmed in gastrointestinal cancers. Pan et al (88) set forth five SNPs (334 $\mathrm{C}>\mathrm{T}, 874 \mathrm{C}>\mathrm{T}, 1,396 \mathrm{G}>\mathrm{A}, 1,690 \mathrm{C}>\mathrm{T}$ and 1,942 T>G) and frameshift mutations in Axin1 involved in GC, in the meantime, a highly mutable $\mathrm{G}$ mononucleotide repeat sequence on exon 7 of Axin 2 continually had a frameshift mutation (1-bp deletion) in gastric cancer with microsatellite instability (MSI) and nuclear $\beta$-catenin stabilization (89). Thus, devoting our efforts to the regulation on $\mathrm{Wnt} / \beta$-catenin pathway in GC will notably benefit anti-metastatic approaches in the clinic.

\section{Interaction between EMT and Wnt/ $\beta$-catenin signaling in gastric cancer}

E-cadherin plays a crucial role in negatively regulating Wnt signaling in addition to the proverbial ingredients of $\beta$-catenin degradation complex. $\beta$-catenin makes up a direct contact between cadherins and $\alpha$-catenin that interacts with the actin cytoskeleton to create tight cell-cell junctions. Accumulating testimony of the significance of cadherin in catenin-mediated cell adherence junctions has confirmed that cytoplasmic carboxyl-terminus of E-cadherin could bind to $\beta$-catenin so as to suppress its nuclear localization (6). On the contrary, Howard et al (90) reported that the capability of $\beta$-catenin to combine with cadherins is necessary for its transcriptional activity since that cadherins might stabilize $\beta$-catenin at membrane by outcompeting its degradation machinery during EMT. Collectively, it appears that crosstalk between cadherins and $\beta$-catenin may be responsible to the process of epithelial-mesenchymal transition. Czyzewska et al (91) proved a statistical connection between not only invasion depth and abnormal $\beta$-catenin expression but also postoperative survival time and expression of E-cadherin. Additionally, notable significance was observed between E-cadherin along with $\beta$-catenin expression in both main mass of tumor and lymph nodes involved. Moreover, Yoshii et al (92) detected that combined loss of membranous E-cadherin and $\beta$-catenin expression in original mass remarkably correlates to lymph node diversion in patients with intestinal type early gastric cancer. It is noteworthy that, Silva et al (93) studied a total of 515 gastric adenocarcinoma patients and separated them into two groups on the basis of their age. In their series, the young group (age $\leq 40$ years) presented with higher percentage of E-cadherin/ $\beta$-catenin membranous expression compared with the old group (age $>40$ years), which could be attributed to the distinguish between diffuse-type and intestinal-type tumors or the mutual effect between E-cadherin and other proteins of Wnt signaling such as APC, suggesting that young GC patients might advance carcinomas through diversified genetic pathways.

Various metastasis molecules, such as leucine zipper transcription factor-like 1 (LZTFL1) (94), paired-related homeobox 1 (PRRX1) (95), Vestigial-like 4 (VGLL4) (96), high mobility group protein A2 (HMGA2) (97), erythropoietin-producing hepatocellular A2 (EphA2) (98), FAT4 (a member of the cadherin superfamily) (99), have been showed to regulate EMT via modulating nuclear location of $\beta$-catenin or target genes of Wnt signaling in gastric cancer. Secreted frizzled-related protein 1 (SFRP1) is classically considered as a conditioner of Wnt pathway by binding Wnt ligands and its ectopic transcription has eventful clinical relevance in gastric cancer development. Qu et al (100) reported that overexpressed sFRP1 is correlated with EMT induction in GC cells as well as lymph node migration and reduced survival time. Han et al (101) established an EMT model by treating BGC-823 cells with doxorubicin and elaborated upregulation of $\beta$-catenin, LEF1, c-Myc which indicates activated $\beta$-catenin signaling. Thereafter, noticeable reversion of EMT biomarkers was found when applying indomethacin or siRNA to stamp down $\beta$-catenin. Given that $\beta$-catenin is critical to cadherinmediated cell adhesion as the reciprocity between $\beta$-catenin and cadherin could promote EMT in gastric cancer, Zhao et al (102) assessed luciferase reporter activity of BGC823 cells where theoretical binding site of $\beta$-catenin on the Vimentin promoter was knocked down by mutagenesis, as a result, Vimentin transcription failed to be regulated. Thus, we may safely draw a conclusion that intervention of EMT progression could be achieved only if $\beta$-catenin operates steadily. Another member of collagen genes, the collagen type I (COL1) was investigated to enhance GC cell motility by means of prompting disconnection of the E-cadherin/catenin compound. Moreover, researchers declared that the dissociation of $\beta$-catenin from E-cadherin and actin cytoskeleton is attributable to its tyrosine phosphorylation, which modifies its chemical attraction to cadherins and induces $\beta$-catenin nuclear trans-situation (103).

Besides, complementary affiliation between EMT and Wnt pathway in regulation of gastric cancer can be referred to as microRNA-dependent post-transcriptional modification. Past evidence is suggestive of the concern of certain miRNAs in different phases of EMT through Wnt signaling and elaboration of which might furnish novel diagnostic or therapeutic options for human gastric cancer hereafter.

\section{MicroRNA-mediated EMT regulatory network in gastric cancer through Wnt pathway}

MicroRNAs have been reported either as anomalously expressed or mutated in various tumors. Specifically, above half of miRNAs locate on tumor associated genomic regions. Analysis of expression patterns of various miRNAs in different gastric cancer tissues has showed the diversity of particular miRNA expression in tumor mass relative to normal mucus. Abundant evidence has verified that a number of miRNAs act as oncogenes or anti-oncogenes while taking part in controlling EMT through targeting at constituents of Wnt pathway (Table I), which indicats the intimate connection between GC evolution and miRNAs.

MicroRNA-200 family comprises five members including miR-200a, miR-200b, miR-200c, miR-141, miR-429 and this family has been extensively acknowledged to be representative of regulatory factors of EMT by means of terminating Wnt $/ \beta$-catenin pathway in gastric cancer. In an effort to determine the suppressive role of miR-200a in gastric cancer, $\mathrm{Su}$ and colleagues (104) employed TOP/FOP flash luciferase assay to elucidate the collaboration beiween miR-200a and Wnt signaling in influencing metastasis potency on GC cells. As a result, they displayed an inverse relationship between miR-200a expression and luciferase activity of CTNNB1, indicating that $\beta$-catenin might be a direct target of miR-200a. 
Table I. MicroRNAs targeting Wnt//-catenin pathway in regulating EMT of GC.

\begin{tabular}{llcc}
\hline MicroRNA & \multicolumn{1}{c}{ Target(s) of Wnt signaling } & Promoter/inhibitor of EMT & Refs. \\
\hline miR-200a & $\beta$-catenin/E-cadherin & Inhibitor & $(104)$ \\
miR-200b & ZEB1/ZEB2/ E-cadherin /Wnt-1 & Inhibitor & $(106-108)$ \\
miR-27 & APC & Promoter & $(109)$ \\
miR-199a-5p & CDH1/3-catenin & Promoter & $(111)$ \\
miR-544a & CDH1/AXIN2 & Promoter & $(112)$ \\
miR-145 & CTNND1 & Inhibitor & \\
\hline
\end{tabular}

Another mechanism by which miR-200a hinders EMT as Cong et al (105) explained was that miR-200a facilitates E-cadherin through upregulating its transcriptional antagonists ZEB1/2 and impacting E-cadherin $/ \beta$-catenin productive competence. Besides, negative correlation of miR-200a to WHO grades as well as EMT proteins via Wnt/ $\beta$-catenin pathway was demonstrated in gastric adenocarcinoma. Furthermore, a cohort of 90 gastric cancer cases was investigated by Song et al (106) to ascertain two microRNA subtypes in accordance with distinct prognosis and the poor-prognosis subtype was identified to possess deregulation of EMT markers. A further identification of the contribution of three pivotal miRNAs (miR-200a, miR-200b, and miR-125b) to poor survival by targeting EMT gene network was conducted while miR-200b was peculiarly confirmed to be able to confront ZEB1 and strengthen E-cadherin in vitro. miR-200b was also validated to act on the 3'UTR of ZEB2 mRNA to antagonize it and localize E-cadherin to the plasma membrane (107). Tang et al (108) explored obvious loss of miR-200b expression in five gastric cancer cells (HGC-27, AGS, MGC-803, BGC-823, SGC-7901 and MKN-28) as well as 27 gastric cancer tissues by qRT-PCR, and the $3^{\prime}$ UTR of Wnt-1 was confirmed to contain the binding regions of miR-200b and they showed restraining expression of Wnt1, $\beta$-catenin and TCF-4 in MGC-803 transfected with miR-200b mimics.

Many other microRNAs have been implied to be significant in modulating EMT through Wnt signaling in GC over and above miR-200 family. Zhang et al (109) recently introduced miR-27 as GC promoter on account of its incremental level in gastric cancer specimens. They stated that overexpressing miR-27 brings about increased ZEB1, ZEB2, Slug, and Vimentin as well as decreased E-cadherin expression level. APC was eventually identified as the immediate target of miR-27 that in turn facilitated Wnt/ $\beta$-catenin pathway and EMT. Serum response factor (SRF) functions as a prometastatic factor in the context of stomach cancer formation, current research performed by Zhao et al (110) provided proof that miR-199a-5p is transactivated when SRF binds to CArG elements in its promoter. The authors reported that exceptional activation of miR-199a-5p leads to not only inhibition of $\mathrm{CDH} 1$ at posttranscriptional level but also import of $\beta$-catenin from adherens junctions to the cytoplasm and nucleus, forcefully indicating that the adjustive effect of miR-199a-5p on EMT may be based on Wnt signaling in GC. miR-544a potentially conferred a role during EMT abduction in gastric cancer cell line, Yanaka and colleagues (111) verified it by way of transfecting MKN1 with a cell-based reporter system which comprises a promoter sequence of Vim. Deeper investigation made clear that miR-544a alters expression of Snail and ZEB1 by directly aiming at the 3'UTR in CDH1 and AXIN2 which successively accelerates nuclear shift of $\beta$-catenin. Xing et al (112) furnished insight into poor clinicopathological parameters arisen from catenin- $\delta 1$ (CTNND1) perversion by inspecting 126 human GC samples utilizing immunohistochemistry. Since Wnt signaling assumes one of the downstream effectors of CTNND1, luciferase assay was implemented to identify the complementariness of miR-145 to the 3'UTR element of CTNND1 mRNA, where enables the diversion of CTNND1-E-cadherin complex from cytoplasm to membrane by downregulating $\mathrm{N}$-cadherin. To sum up, microRNAs keep an intimate connection with EMT and Wnt related genes in gastric cancer as the key regulatory factors in post-transcription network. Clarification of the participation of miRNAs in reversing EMT process may exert profound influence on gastric cancer diagnosis, therapy or prognostic evaluation.

\section{Promising targeted therapy in gastric cancer}

Epithelial-mesenchymal transition has established itself as a foundational process through which epithelial cells turn into mesenchymal accompanied by apparent genetic and phenotypic modifications. Assertive evidence is mounting that $\mathrm{Wnt} / \beta$-catenin signaling is one of the considerable pathways orchestrating EMT mainly due to the catenin/cadherin complex. Owing to the mutuality among EMT and Wnt/ $\beta$ catenin pathway in GC pathogenesis, researchers are making efforts to excavate potential molecules as therapeutic targets for more precise treatment.

The Runt-domain transcription factor 3 (Runx3) has a defending effect on gastric epithelium as it mediates cellular proliferation and apoptosis while as many as $80 \%$ of GC patients are observed to carry loss of Runx3. Gastric epithelial cells become more plastic and susceptible to spontaneous EMT in absence of Runx 3 and perverse Wnt signaling fuels the immortal cells to become carcinogenic, while both could be abolished by endogenous Runx3 inversely, signifying that Runx 3 could be deemed as a capable treatment targets (113). Sox10 is a member of the SRY-related-HMG-box family that has been documented to be silenced by promoter methylation in GC while its overexpression remarkably attenuates distinct organ metastasis in nude mice. Mechanistically, competition with TCF4 for $\beta$-catenin binding consensus element is required for the EMT restraint of Sox 10 (114). Cai et al (99) 
examined the repressive role of FAT4, a cadherin-associated protein, in gastric cancer and the underlying theory. Knocking out FAT4 boosted pulmonary and hepatic metastatic lesions in vivo as well as the upregulation of EMT biomarkers in vitro. Notably, this tumorous promotion was abrogated on the condition of siRNA-mediated silencing of $\beta$-catenin. Furthermore, many other molecules serve as agitators via motivating Wnt $\beta$ catenin pathway durung GC progress. EphA2 is a member of the RTK family whose upregulated level is usually connected to higher aggressiveness in gastric cancer. Huang et al (98) found that overexpression of EphA2 contributes to upregulation of N-cadherin and Snail while knockdown of EphA2 elicits inverted effect. More significantly, the result of EphA2 enhancement was neutralized when applying XAV939 to terminate Wnt signaling, whereas agitation of Wnt pathway by $\mathrm{LiCl}$ remedied the influence of EphA2 silencing on EMT in the GC cell lines AGS and SGC7901. Zha et al (97) verified the oncogenic character of HMGA2 in nude mice subcutaneously transplanted with MKN-28 and further study conclusively elucidated HMGA2 as a contributor to EMT by preventing $\beta$-catenin from phosphorylation degradationin in order to activate Wnt signaling. Analogously, PRRX1 was proven to be positively related to EMT and Wnt/ $\beta$-catenin signaling downstream the molecule markers in GC specimens, while the reinforced EMT and cell mobility was offset by inhibition of Wnt pathway in BGC823 and SGC7901 lentiviral overexpression of PRRX1 (95). Since a great number of similar molecules are playing a part in EMT through modulating Wnt/ $\beta$-catenin pathway during GC progression, further insight into directing these potential targets to reverse EMT can be applicable to gastric cancer clinically.

\section{Conclusions and perspectives}

In brief, numerous potential molecules act the part of inducers or suppressors of EMT by coordinating Wnt/ $\beta$-catenin pathway in gastric cancer. MicroRNAs at the same time serve as a link between EMT and Wnt signaling in human GC and miRNAbased therapeutics such as antisense oligonucleotides, Locked nucleic acid (LNA) antimiR constructs, miRNAs Sponges and miR-Mask, have been investigated and developed (115). Nonetheless, elaborate pharmacodynamic and pharmacokinetic investigation in vivo models as well as rigorous clinical trials in GC patients are urged to eliminate off target side effects and ensure regional effective concentration as far as possible. We firmly believe that reformative understanding of the impact of related molecules on catenin/cadherin compound would contribute to advanced therapeutical applications and better prognosis for human gastric cancer.

\section{Acknowledgements}

This study was supported by the National Natural Science Foundation of China (no. 81473605), State Administration of Traditional Chinese Medicine of the People's Republic of China (JDZX2012087), the Special Grants for Leading Principal Investigators from Jiangsu Administration of Traditional Chinese Medicine (LJ200908), Key Discipline Construction Program from Jiangsu Administration of Traditional Chinese Medicine (JS1305), Jiangsu Provincial
Commission of Health and Family Planning (BJ14013), the Priority Academic Program Development of Jiangsu Higher Education Institutions (PAPD), Jiangsu Provincial Special Program of Medical Science (BL2014100), and Scientific Research Innovation for Graduates from Jiangsu Higher Education Institutions (SJLX15_0440).

\section{References}

1. Guimarães RM and Muzi CD: Trend of mortality rates for gastric cancer in Brazil and regions in the period of 30 years (1980-2009). Arq Gastroenterol 49: 184-188, 2012.

2. Ferlay J, Shin HR, Bray F, Forman D, Mathers C and Parkin DM: Estimates of worldwide burden of cancer in 2008: GLOBOCAN 2008. Int J Cancer 127: 2893-2917, 2010.

3. Torre LA, Bray F, Siegel RL, Ferlay J, Lortet-Tieulent J and Jemal A: Global cancer statistics, 2012. CA Cancer J Clin 65: 87-108, 2015

4. Shamir ER, Pappalardo E, Jorgens DM, Coutinho K, Tsai WT, Aziz K, Auer M, Tran PT, Bader JS and Ewald AJ: Twist1induced dissemination preserves epithelial identity and requires E-cadherin. J Cell Biol 204: 839-856, 2014.

5. Pasquier J, Abu-Kaoud N, Al Thani H and Rafii A: Epithelial to mesenchymal transition in a clinical perspective. J Oncol 2015: 792182, 2015.

6. Heuberger J and Birchmeier W: Interplay of cadherin-mediated cell adhesion and canonical Wnt signaling. Cold Spring Harb Perspect Biol 2: a002915, 2010.

7. Zhao JH, Luo Y, Jiang YG, He DL and Wu CT: Knockdown of $\beta$-catenin through shRNA cause a reversal of EMT and metastatic phenotypes induced by HIF-1 $\alpha$. Cancer Invest 29: 377-382, 2011.

8. Chang YW, Su YJ, Hsiao M, Wei KC, Lin WH, Liang CL, Chen SC and Lee JL: Diverse targets of $\beta$-catenin during the epithelial-mesenchymal transition define cancer stem cells and predict disease relapse. Cancer Res 75: 3398-3410, 2015.

9. Moyret-Lalle C, Ruiz E and Puisieux A: Epithelial-mesenchymal transition transcription factors and miRNAs: 'Plastic surgeons' of breast cancer. World J Clin Oncol 5: 311-322, 2014.

10. Greenburg G and Hay ED: Epithelia suspended in collagen gels can lose polarity and express characteristics of migrating mesenchymal cells. J Cell Biol 95: 333-339, 1982.

11. Steinestel K, Eder S, Schrader AJ and Steinestel J: Clinical significance of epithelial-mesenchymal transition. Clin Transl Med 3: 17, 2014.

12. Costabile V, Duraturo F, Delrio P, Rega D, Pace U, Liccardo R, Rossi GB, Genesio R, Nitsch L, Izzo P, et al: Lithium chloride induces mesenchymal-to-epithelial reverting transition in primary colon cancer cell cultures. Int J Oncol 46: 1913-1923, 2015.

13. Logullo AF, Nonogaki S, Pasini FS, Osório CA, Soares FA and Brentani MM: Concomitant expression of epithelialmesenchymal transition biomarkers in breast ductal carcinoma: Association with progression. Oncol Rep 23: 313-320, 2010.

14. Nozato M, Kaneko S, Nakagawara A and Komuro H: Epithelialmesenchymal transition-related gene expression as a new prognostic marker for neuroblastoma. Int J Oncol 42: 134-140, 2013.

15. Nilsson GM, Akhtar N, Kannius-Janson M and Baeckström D: Loss of E-cadherin expression is not a prerequisite for c-erbB2induced epithelial-mesenchymal transition. Int J Oncol 45: 82-94, 2014.

16. Moghbeli M, Moaven O, Memar B, Raziei HR, Aarabi A, Dadkhah E, Forghanifard MM, ManzariF and Abbaszadegan MR: Role of hMLH1 and E-cadherin promoter methylation in gastric cancer progression. J Gastrointest Cancer 45: 40-47, 2014.

17. Yu QM, Wang XB, Luo J, Wang S, Fang XH, Yu JL and Ling ZQ: $\mathrm{CDH} 1$ methylation in preoperative peritoneal washes is an independent prognostic factor for gastric cancer. J Surg Oncol 106: 765-771, 2012.

18. Hansford S, Kaurah P, Li-Chang H, Woo M, Senz J, Pinheiro H, Schrader KA, Schaeffer DF, Shumansky K, Zogopoulos G, et al: Hereditary diffuse gastric cancer syndrome: CDH1 mutations and beyond. JAMA Oncol 1: 23-32, 2015.

19. Li XJ, Zhao Y and Ren H: E-cadherin expression and CDH1 promoter methylation in sporadic and hereditary gastric cancer]. Nan Fang Yi Ke Da Xue Xue Bao 35: 125-127, 2015 (In Chinese). 
20. Jun KH, Lee JE, Kim SH, Jung JH, Choi HJ, Kim YI, Chin HM and Yang SH: Clinicopathological significance of N-cadherin and VEGF in advanced gastric cancer brain metastasis and the effects of metformin in preclinical models. Oncol Rep 34: 2047-2053, 2015.

21. Kamikihara $\mathrm{T}$, Ishigami $\mathrm{S}$, Arigami $\mathrm{T}$, Matsumoto $\mathrm{M}$, Okumura H, Uchikado Y, Kita Y, Kurahara H, Kijima Y, Ueno S, et al: Clinical implications of $\mathrm{N}$-cadherin expression in gastric cancer. Pathol Int 62: 161-166, 2012.

22. Otsuki S, Inokuchi M, Enjoji M, Ishikawa T, Takagi Y, Kato K, Yamada $\mathrm{H}$, Kojima $\mathrm{K}$ and Sugihara K: Vimentin expression is associated with decreased survival in gastric cancer. Oncol Rep 25: 1235-1242, 2011

23. Shirahata A, Sakuraba K, Kitamura Y, Yokomizo K, Gotou T, Saitou M, Kigawa G, Nemoto H, Sanada Y and Hibi K: Detection of vimentin methylation in the serum of patients with gastric cancer. Anticancer Res 32: 791-794, 2012.

24. Zeisberg M and Neilson EG: Biomarkers for epithelial-mesenchymal transitions. J Clin Invest 119: 1429-1437, 2009.

25. Hu Z, Liu X, Tang Z, Zhou Y and Qiao L: Possible regulatory role of Snail in NF- $\mathrm{KB}$-mediated changes in E-cadherin in gastric cancer. Oncol Rep 29: 993-1000, 2013.

26. Zhang J, Zhou Y and Yang Y: CCR7 pathway induces epithelialmesenchymal transition through up-regulation of Snail signaling in gastric cancer. Med Oncol 32: 467, 2015.

27. Shin NR, Jeong EH, Choi CI, Moon HJ, Kwon CH, Chu IS, Kim GH, Jeon TY, Kim DH, Lee JH, et al: Overexpression of Snail is associated with lymph node metastasis and poor prognosis in patients with gastric cancer. BMC Cancer 12: 521, 2012

28. Barnes RM and Firulli AB: A twist of insight - the role of Twistfamily bHLH factors in development. Int J Dev Biol 53: 909-924, 2009.

29. Liu AN, Zhu ZH, Chang SJ and Hang XS: Twist expression associated with the epithelial-mesenchymal transition in gastric cancer. Mol Cell Biochem 367: 195-203, 2012.

30. Ru GQ, Wang HJ, Xu WJ and Zhao ZS: Upregulation of Twist in gastric carcinoma associated with tumor invasion and poor prognosis. Pathol Oncol Res 17: 341-347, 2011.

31. Qian J, Luo Y, Gu X, Zhan W and Wang X: Twist1 promotes gastric cancer cell proliferation through up-regulation of FoxM1. PLoS One 8: e77625, 2013.

32. Zhang H, Gong J, Kong D and Liu HY: Anti-proliferation effects of Twist gene silencing in gastric cancer SGC7901 cells. World J Gastroenterol 21: 2926-2936, 2015.

33. Choi YJ, Kim N, Chang H, Lee HS, Park SM, Park JH, Shin CM, Kim JM, Kim JS, Lee DH, et al: Helicobacter pylori-induced epithelial-mesenchymal transition, a potential role of gastric cancer initiation and an emergence of stem cells. Carcinogenesis 36: 553-563, 2015.

34. Okugawa Y, Toiyama Y, Tanaka K, Matsusita K, Fujikawa H, Saigusa S, Ohi M, Inoue Y, Mohri Y, Uchida K, et al: Clinical significance of Zinc finger E-box Binding homeobox 1 (ZEB1) in human gastric cancer. J Surg Oncol 106: 280-285, 2012

35. Yabusaki N, Yamada S, Murai T, Kanda M, Kobayashi D, Tanaka C, Fujii T, Nakayama G, Sugimoto H, Koike M, et al Clinical significance of zinc-finger E-box binding homeobox 1 mRNA levels in peritoneal washing for gastric cancer. Mol Clin Oncol 3: 435-441, 2015.

36. Murai T, Yamada S, Fuchs BC, Fujii T, Nakayama G, Sugimoto H, Koike M, Fujiwara M, Tanabe KK and Kodera Y: Epithelial-tomesenchymal transition predicts prognosis in clinical gastric cancer. J Surg Oncol 109: 684-689, 2014.

37. Jia B, Liu H, Kong Q and Li B: Overexpression of ZEB1 associated with metastasis and invasion in patients with gastric carcinoma. Mol Cell Biochem 366: 223-229, 2012

38. Dai YH, Tang YP, Zhu HY, Lv L, Chu Y, Zhou YQ and Huo JR: ZEB2 promotes the metastasis of gastric cancer and modulates epithelial mesenchymal transition of gastric cancer cells. Dig Dis Sci 57: 1253-1260, 2012.

39. Minn YK, Lee H, Hyung WJ, Kim JE, Choi J, Yang SH, Song H, Lim BJ and Kim SH: MicroRNA-200 family members and ZEB2 are associated with brain metastasis in gastric adenocarcinoma. Int J Oncol 45: 2403-2410, 2014.

40. Brabletz T: To differentiate or not - routes towards metastasis. Nat Rev Cancer 12: 425-436, 2012.

41. Reya T, Morrison SJ, Clarke MF and Weissman IL: Stem cells, cancer, and cancer stem cells. Nature 414: 105-111, 2001.

42. Zhang C, Li C, He F, Cai Y and Yang H: Identification of $\mathrm{CD} 44^{+} \mathrm{CD} 24^{+}$gastric cancer stem cells. J Cancer Res Clin Oncol 137: 1679-1686, 2011
43. Han ME, Jeon TY, Hwang SH, Lee YS, Kim HJ, Shim HE, Yoon S, Baek SY, Kim BS, Kang CD, et al: Cancer spheres from gastric cancer patients provide an ideal model system for cancer stem cell research. Cell Mol Life Sci 68: 3589-3605, 2011.

44. Jiang J, Zhang Y, Chuai S, Wang Z, Zheng D, Xu F, Zhang Y, Li C, Liang Y and Chen Z: Trastuzumab (herceptin) targets gastric cancer stem cells characterized by CD90 phenotype. Oncogene 31: 671-682, 2012

45. Chen T, Yang K, Yu J, Meng W, Yuan D, Bi F, Liu F, Liu J, Dai B, Chen $\mathrm{X}$, et al: Identification and expansion of cancer stem cells in tumor tissues and peripheral blood derived from gastric adenocarcinoma patients. Cell Res 22: 248-258, 2012.

46. Yoshida K, Tsujimoto H, Matsumura K, Kinoshita M, Takahata R, Matsumoto Y, Hiraki S, Ono S, Seki S, Yamamoto J, et al: CD47 is an adverse prognostic factor and a therapeutic target in gastric cancer. Cancer Med 4: 1322-1333, 2015

47. Yang L, Ping YF, Yu X, Qian F, Guo ZJ, Qian C, Cui YH and Bian XW: Gastric cancer stem-like cells possess higher capability of invasion and metastasis in association with a mesenchymal transition phenotype. Cancer Lett 310: 46-52, 2011.

48. Bessède E, Staedel C, Acuña Amador LA, Nguyen PH, Chambonnier L, Hatakeyama M, Belleannée G, Mégraud F and Varon C: Helicobacter pylori generates cells with cancer stem cell properties via epithelial-mesenchymal transition-like changes. Oncogene 33: 4123-4131, 2014.

49. Ryu HS, Park J, Kim HH, Kim WH and Lee HS: Combination of epithelial-mesenchymal transition and cancer stem cell-like phenotypes has independent prognostic value in gastric cancer. Hum Pathol 43: 520-528, 2012.

50. Xu GF, Zhang WJ, Sun Q, Xu X, Zou X and Guan W: Combined epithelial-mesenchymal transition with cancer stem cell-like marker as predictors of recurrence after radical resection for gastric cancer. World J Surg Oncol 12: 368, 2014.

51. Jang YS, Sim JJ, Ji E, Jeong KY and Kim HM: Investigation of lactate calcium salt-induced $\beta$-catenin destabilization in colorectal cancer cells. Life Sci 139: 160-165, 2015.

52. Liu X, Yun F, Shi L, Li ZH, Luo NR and Jia YF: Roles of signaling pathways in the epithelial-mesenchymal transition in cancer. Asian Pac J Cancer Prev 16: 6201-6206, 2015.

53. Zhang $X$ and Hao J: Development of anticancer agents targeting the Wnt/ $\beta$-catenin signaling. Am J Cancer Res 5: 2344-2360, 2015.

54. Yoshida GJ and Saya H: Inversed relationship between CD44 variant and c-Myc due to oxidative stress-induced canonical Wnt activation. Biochem Biophys Res Commun 443: 622-627, 2014.

55. Mao J, Fan S, Ma W, Fan P, Wang B, Zhang J, Wang H, Tang B, Zhang Q, Yu X, et al: Roles of Wnt/ $\beta$-catenin signaling in the gastric cancer stem cells proliferation and salinomycin treatment. Cell Death Dis 5: e1039, 2014.

56. Zhang $\mathrm{H}$ and Xue $\mathrm{Y}$ : Wnt pathway is involved in advanced gastric carcinoma. Hepatogastroenterology 55: 1126-1130, 2008.

57. Cheng XX, Wang ZC, Chen XY, Sun Y, Kong QY, Liu J and $\mathrm{Li} \mathrm{H}$ : Correlation of Wnt-2 expression and beta-catenin intracellular accumulation in Chinese gastric cancers: Relevance with tumour dissemination. Cancer Lett 223: 339-347, 2005.

58. Kurayoshi M, Oue N, Yamamoto $\mathrm{H}$, Kishida M, Inoue A, Asahara T, Yasui W and Kikuchi A: Expression of Wnt-5a is correlated with aggressiveness of gastric cancer by stimulating cell migration and invasion. Cancer Res 66: 10439-10448, 2006.

59. Kirikoshi H, Sekihara H and Katoh M: Up-regulation of WNT10A by tumor necrosis factor alpha and Helicobacter pylori in gastric cancer. Int J Oncol 19: 533-536, 2001.

60. Saitoh T, Kirikoshi H, Mine T and Katoh M: Proto-oncogene WNT10B is up-regulated by tumor necrosis factor alpha in human gastric cancer cell line MKN45. Int J Oncol 19: 1187-1192, 2001.

61. Figueiredo J, Söderberg O, Simões-Correia J, Grannas K, Suriano G and Seruca R: The importance of E-cadherin binding partners to evaluate the pathogenicity of E-cadherin missense mutations associated to HDGC. Eur J Hum Genet 21: 301-309, 2013.

62. Garziera M, Canzonieri V, Cannizzaro R, Geremia S, Caggiari L, De Zorzi M, Maiero S, Orzes E, Perin T, Zanussi S, et al: Identification and characterization of $\mathrm{CDH} 1$ germline variants in sporadic gastric cancer patients and in individuals at risk of gastric cancer. PLoS One 8: e77035, 2013.

63. Humar B, Blair V, Charlton A, More H, Martin I and Guilford P. E-cadherin deficiency initiates gastric signet-ring cell carcinoma in mice and man. Cancer Res 69: 2050-2056, 2009. 
64. Humar B and Guilford P: Hereditary diffuse gastric cancer: A manifestation of lost cell polarity. Cancer Sci 100: 1151-1157, 2009.

65. Mutoh H, Sakurai S, Satoh K, Tamada K, Kita H, Osawa H, Tomiyama T, Sato Y, Yamamoto H, Isoda N, et al: Development of gastric carcinoma from intestinal metaplasia in $\mathrm{Cdx} 2$ transgenic mice. Cancer Res 64: 7740-7747, 2004.

66. Kim HS, Lee JS, Freund JN, Min KW, Lee JS, Kim W, Juhng SW and Park CS: CDX-2 homeobox gene expression in human gastric carcinoma and precursor lesions. J Gastroenterol Hepatol 21: 438-442, 2006

67. Clements WM, Wang J, Sarnaik A, Kim OJ, MacDonald J, Fenoglio-Preiser C, Groden J and Lowy AM: $\beta$-catenin mutation is a frequent cause of Wnt pathway activation in gastric cancer. Cancer Res 62: 3503-3506, 2002.

68. Yoda Y, Takeshima H, Niwa T, Kim JG, Ando T, Kushima R, Sugiyama T, Katai H, Noshiro H and Ushijima T: Integrated analysis of cancer-related pathways affected by genetic and epigenetic alterations in gastric cancer. Gastric Cancer 18: 65-76, 2015.

69. Kim SJ, Shin JY, Cheong TC, Choi IJ, Lee YS, Park SH and Chun KH: Galectin-3 germline variant at position 191 enhances nuclear accumulation and activation of $\beta$-catenin in gastric cancer. Clin Exp Metastasis 28: 743-750, 2011.

70. Koo BK, Spit M, Jordens I, Low TY, Stange DE, van de Wetering M, van Es JH, Mohammed S, Heck AJ, Maurice MM, et al: Tumour suppressor RNF43 is a stem-cell E3 ligase that induces endocytosis of Wnt receptors. Nature 488: 665-669, 2012.

71. Hao HX, Xie Y, Zhang Y, Charlat O, Oster E, Avello M, Lei H, Mickanin C, Liu D, Ruffner H, et al: ZNRF3 promotes Wnt receptor turnover in an R-spondin-sensitive manner. Nature 485: 195-200, 2012

72. Jo YS, Kim MS, Lee JH, Lee SH, An CH and Yoo NJ: Frequent frameshift mutations in 2 mononucleotide repeats of RNF43 gene and its regional heterogeneity in gastric and colorectal cancers. Hum Pathol 46: 1640-1646, 2015.

73. Lu H, Sun J, Wang F, Feng L, Ma Y, Shen Q, Jiang Z, Sun X, Wang $\mathrm{X}$ and Jin $\mathrm{H}$ : Enhancer of zeste homolog 2 activates wnt signaling through downregulating CXXC finger protein 4. Cell Death Dis 4: e776, 2013.

74. Pandi NS, Manimuthu M, Harunipriya P, Murugesan M, Asha GV and Rajendran S: In silico analysis of expression pattern of a Wnt $/ \beta$-catenin responsive gene ANLN in gastric cancer. Gene 545: 23-29, 2014

75. Qiu HB, Zhang LY, Ren C, Zeng ZL, Wu WJ, Luo HY, Zhou ZW and Xu RH: Targeting CDH17 suppresses tumor progression in gastric cancer by downregulating Wnt/ $\beta$-catenin signaling. PLoS One 8: e56959, 2013.

76. To KF, Chan MW, Leung WK, Yu J, Tong JH, Lee TL, Chan FK and Sung JJ: Alterations of frizzled (FzE3) and secreted frizzled related protein (hsFRP) expression in gastric cancer. Life Sci 70: 483-489, 2001

77. Kang W, Tong JH, Chan AW, Zhao J, Dong Y, Wang S, Yang W, Sin FM, Ng SS, Yu J, et al: Yin Yang 1 contributes to gastric carcinogenesis and its nuclear expression correlates with shorter survival in patients with early stage gastric adenocarcinoma. J Transl Med 12: 80, 2014.

78. Mo ML, Li MR, Chen Z, Liu XW, Sheng Q and Zhou HM: Inhibition of the Wnt palmitoyltransferase porcupine suppresses cell growth and downregulates the Wnt/ $\beta$-catenin pathway in gastric cancer. Oncol Lett 5: 1719-1723, 2013.

79. Cai $C$ and Zhu $X$ : The Wnt/ $\beta$-catenin pathway regulates selfrenewal of cancer stem-like cells in human gastric cancer. Mol Med Rep 5: 1191-1196, 2012.

80. Yu J, Tao Q, Cheng YY, Lee KY, Ng SS, Cheung KF, Tian L, Rha SY, Neumann U, Röcken C, et al: Promoter methylation of the Wnt/beta-catenin signaling antagonist Dkk-3 is associated with poor survival in gastric cancer. Cancer 115: 49-60, 2009.

81. Than SS, Kataoka K, Sakaguchi M, Murata H, Abarzua F, Taketa C, Du G, Yashiro M, Yanagihara K, Nasu Y, et al: Intraperitoneal administration of an adenovirus vector carrying REIC/Dkk-3 suppresses peritoneal dissemination of scirrhous gastric carcinoma. Oncol Rep 25: 989-995, 2011.

82. Gomceli I, Bostanci EB, Ozer I, Kemik AS, Turhan N, Tez M, Kilic S, Demiriz B and Akoglu M: A novel screening biomarker in gastric cancer: Serum Dickkopf-1. Hepatogastroenterology 59 1661-1664, 2012.
83. Mühlmann G, Untergasser G, Zitt M, Zitt M, Maier H, Mikuz G, Kronberger IE, Haffner MC, Gunsilius E and Ofner D: Immunohistochemically detectable dickkopf-3 expression in tumor vessels predicts survival in gastric cancer. Virchows Arch 456: 635-646, 2010.

84. Hu Y, Wan R, Yu G, Shen J, Ni J, Yin G, Xing M, Chen C, Fan Y, Xiao W, et al: Imbalance of Wnt/Dkk negative feedback promotes persistent activation of pancreatic stellate cells in chronic pancreatitis. PLoS One 9: e95145, 2014

85. Shin H, Kim JH, Lee YS and Lee YC: Change in gene expression profiles of secreted frizzled-related proteins (SFRPs) by sodium butyrate in gastric cancers: Induction of promoter demethylation and histone modification causing inhibition of Wnt signaling. Int J Oncol 40: 1533-1542, 2012.

86. Ebert MP, Fei G, Kahmann S, Müller O, Yu J, Sung JJ and Malfertheiner P: Increased $\beta$-catenin mRNA levels and mutational alterations of the APC and $\beta$-catenin gene are present in intestinal-type gastric cancer. Carcinogenesis 23: 87-91, 2002.

87. Fang Z, Xiong Y, Li J, Liu L, Zhang W, Zhang C and Wan J: APC gene deletions in gastric adenocarcinomas in a Chinese population: A correlation with tumour progression. Clin Transl Oncol 14: 60-65, 2012.

88. Pan KF, Liu WG, Zhang L, You WC and Lu YY: Mutations in components of the Wnt signaling pathway in gastric cancer. World J Gastroenterol 14: 1570-1574, 2008.

89. Mazzoni SM and Fearon ER: AXIN1 and AXIN2 variants in gastrointestinal cancers. Cancer Lett 355: 1-8, 2014.

90. Howard S, Deroo T, Fujita Y and Itasaki N: A positive role of cadherin in Wnt/ $\beta$-catenin signalling during epithelial-mesenchymal transition. PLoS One 6: e23899, 2011.

91. Czyzewska J, Guzińska-Ustymowicz K, Ustymowicz M, Pryczynicz A and Kemona A: The expression of E-cadherincatenin complex in patients with advanced gastric cancer: Role in formation of metastasis. Folia Histochem Cytobiol 48: 37-45, 2010.

92. Yoshii T, Miyagi Y, Nakamura Y, Kobayashi O, Kameda Y and Ohkawa S: Pilot research for the correlation between the expression pattern of E-cadherin- $\beta$-catenin complex and lymph node metastasis in early gastric cancer. Tumori 99: 234-238, 2013.

93. Silva EM, Begnami MD, Fregnani JH, Pelosof AG, Zitron C, Montagnini AL and Soares FA: Cadherin-catenin adhesion system and mucin expression: A comparison between young and older patients with gastric carcinoma. Gastric Cancer 11: 149-159, 2008

94. Wang L, Guo J, Wang Q, Zhou J, Xu C, Teng R, Chen Y, Wei Q and Liu ZP: LZTFL1 suppresses gastric cancer cell migration and invasion through regulating nuclear translocation of $\beta$-catenin. J Cancer Res Clin Oncol 140: 1997-2008, 2014.

95. Guo J, Fu Z, Wei J, Lu W, Feng J and Zhang S: PRRX1 promotes epithelial-mesenchymal transition through the Wnt/ $\beta$-catenin pathway in gastric cancer. Med Oncol 32: 393, 2015.

96. Li H, Wang Z, Zhang W, Qian K, Liao G, Xu W and Zhang S: VGLL4 inhibits EMT in part through suppressing Wnt/ $\beta$ catenin signaling pathway in gastric cancer. Med Oncol 32: 83, 2015.

97. Zha L, Zhang J, Tang W, Zhang N, He M, Guo Y and Wang Z: HMGA2 elicits EMT by activating the $\mathrm{Wnt} / \beta$-catenin pathway in gastric cancer. Dig Dis Sci 58: 724-733, 2013.

98. Huang J, Xiao D, Li G, Ma J, Chen P, Yuan W, Hou F, Ge J, Zhong M, Tang Y, et al: EphA2 promotes epithelial-mesenchymal transition through the Wnt/ $\beta$-catenin pathway in gastric cancer cells. Oncogene 33: 2737-2747, 2014.

99. Cai J, Feng D, Hu L, Chen H, Yang G, Cai Q, Gao C and Wei D: FAT4 functions as a tumour suppressor in gastric cancer by modulating Wnt $/ \beta$-catenin signalling. Br J Cancer 113 : 1720-1729, 2015.

100. Qu Y, Ray PS, Li J, Cai Q, Bagaria SP, Moran C, Sim MS, Zhang J, Turner RR, Zhu Z, et al: High levels of secreted frizzled-related protein 1 correlate with poor prognosis and promote tumourigenesis in gastric cancer. Eur J Cancer 49: 3718-3728, 2013.

101. Han R, Xiong J, Xiao R, Altaf E, Wang J, Liu Y, Xu H, Ding Q and Zhang Q: Activation of $\beta$-catenin signaling is critical for doxorubicin-induced epithelial-mesenchymal transition in BGC-823 gastric cancer cell line. Tumour Biol 34: 277-284, 2013.

102. Zhao L, Li W, Zang W, Liu Z, Xu X, Yu H, Yang Q and Jia J: JMJD2B promotes epithelial-mesenchymal transition by cooperating with $\beta$-catenin and enhances gastric cancer metastasis. Clin Cancer Res 19: 6419-6429, 2013. 
103. Li A, Zhou T, Guo L and Si J: Collagen type I regulates $\beta$-catenin tyrosine phosphorylation and nuclear translocation to promote migration and proliferation of gastric carcinoma cells. Oncol Rep 23: 1247-1255, 2010.

104. Su J, Zhang A, Shi Z, Ma F, Pu P, Wang T, Zhang J, Kang C and Zhang Q: MicroRNA-200a suppresses the Wnt/ $\beta$-catenin signaling pathway by interacting with $\beta$-catenin. Int J Oncol 40: 1162-1170, 2012.

105. Cong N, Du P, Zhang A, Shen F, Su J, Pu P, Wang T, Zjang J, Kang $C$ and Zhang Q: Downregulated microRNA-200a promotes EMT and tumor growth through the wnt/ $\beta$-catenin pathway by targeting the E-cadherin repressors ZEB1/ZEB2 in gastric adenocarcinoma. Oncol Rep 29: 1579-1587, 2013.

106. Song F, Yang D, Liu B, Guo Y, Zheng H, Li L, Wang T, Yu J, Zhao Y, Niu R, et al: Integrated microRNA network analyses identify a poor-prognosis subtype of gastric cancer characterized by the miR-200 family. Clin Cancer Res 20: 878-889, 2014.

107. Kurashige J, Kamohara H, Watanabe M, Hiyoshi Y, Iwatsuki M, Tanaka Y, Kinoshita K, Saito S, Baba Y and Baba H MicroRNA-200b regulates cell proliferation, invasion, and migration by directly targeting ZEB2 in gastric carcinoma. Ann Surg Oncol 19 (Suppl 3): S656-S664, 2012.

108. Tang H, Kong Y, Guo J, Tang Y, Xie X, Yang L, Su Q and Xie X: Diallyl disulfide suppresses proliferation and induces apoptosis in human gastric cancer through Wnt-1 signaling pathway by up-regulation of miR-200b and miR-22. Cancer Lett 340: 72-81, 2013.
109. Zhang Z, Liu S, Shi R and Zhao G: miR-27 promotes human gastric cancer cell metastasis by inducing epithelial-to-mesenchymal transition. Cancer Genet 204: 486-491, 2011.

110. Zhao X, He L, Li T, Lu Y, Miao Y, Liang S, Guo H, Bai M, Xie H, Luo G, et al: SRF expedites metastasis and modulates the epithelial to mesenchymal transition by regulating miR199a-5p expression in human gastric cancer. Cell Death Differ 21: 1900-1913, 2014.

111. Yanaka Y, Muramatsu T, Uetake H, Kozaki K and Inazawa J: miR-544a induces epithelial-mesenchymal transition through the activation of WNT signaling pathway in gastric cancer. Carcinogenesis 36: 1363-1371, 2015.

112. Xing AY, Wang YW, Su ZX, Shi DB, Wang B and Gao P. Catenin- $\delta 1$, negatively regulated by miR-145, promotes tumour aggressiveness in gastric cancer. J Pathol 236: 53-64, 2015.

113. Voon DC, Wang H, Koo JK, Nguyen TA, Hor YT, Chu YS, Ito K, Fukamachi H, Chan SL, Thiery JP, et al: Runx3 protects gastric epithelial cells against epithelial-mesenchymal transitioninduced cellular plasticity and tumorigenicity. Stem Cells 30: 2088-2099, 2012.

114. Tong X, Li L, Li X, Heng L, Zhong L, Su X, Rong R, Hu S, Liu W, Jia B, et al: SOX10, a novel HMG-box-containing tumor suppressor, inhibits growth and metastasis of digestive cancers by suppressing the $\mathrm{Wnt} / \beta$-catenin pathway. Oncotarget 5: 10571-10583, 2014

115. Garzon R, Marcucci G and Croce CM: Targeting microRNAs in cancer: Rationale, strategies and challenges. Nat Rev Drug Discov 9: 775-789, 2010. 